\title{
Apelin directs endothelial cell differentiation and vascular repair following immune-mediated injury
}

\author{
Andrew G. Masoud, ${ }^{1}$ Jiaxin Lin, ${ }^{2,3}$ Abul K. Azad, ${ }^{1}$ Maikel A. Farhan, ${ }^{1}$ Conrad Fischer, ${ }^{4}$ Lin F. Zhu, ${ }^{2}$ Hao Zhang, ${ }^{1,5}$ Banu Sis, ${ }^{6}$ \\ Zamaneh Kassiri, ${ }^{7}$ Ronald B. Moore, ${ }^{2}$ Daniel Kim, ${ }^{1}$ Colin C. Anderson, ${ }^{2,3}$ John C. Vederas, ${ }^{4}$ Benjamin A. Adam, ${ }^{6}$ \\ Gavin Y. Oudit, ${ }^{1,5}$ and Allan G. Murray ${ }^{1}$ \\ 1Department of Medicine, ${ }^{2}$ Department of Surgery, ${ }^{3}$ Department of Medical Microbiology and Immunology, and ${ }^{4}$ Department of Chemistry, University of Alberta, Edmonton, Alberta, Canada. ${ }^{5}$ Mazankowski \\ Heart Institute, Edmonton, Alberta, Canada. ${ }^{6}$ Department of Laboratory Medicine and Pathology and ${ }^{7}$ Department of Physiology, University of Alberta, Edmonton, Alberta, Canada.
}

\begin{abstract}
Sustained, indolent immune injury of the vasculature of a heart transplant limits long-term graft and recipient survival. This injury is mitigated by a poorly characterized, maladaptive repair response. Vascular endothelial cells respond to proangiogenic cues in the embryo by differentiation to specialized phenotypes, associated with expression of apelin. In the adult, the role of developmental proangiogenic cues in repair of the established vasculature is largely unknown. We found that human and minor histocompatibility-mismatched donor mouse heart allografts with alloimmune-mediated vasculopathy upregulated expression of apelin in arteries and myocardial microvessels. In vivo, loss of donor heart expression of apelin facilitated graft immune cell infiltration, blunted vascular repair, and worsened occlusive vasculopathy in mice. In vitro, an apelin receptor agonist analog elicited endothelial nitric oxide synthase activation to promote endothelial monolayer wound repair and reduce immune cell adhesion. Thus, apelin acted as an autocrine growth cue to sustain vascular repair and mitigate the effects of immune injury. Treatment with an apelin receptor agonist after vasculopathy was established markedly reduced progression of arterial occlusion in mice. Together, these initial data identify proangiogenic apelin as a key mediator of coronary vascular repair and a pharmacotherapeutic target for immune-mediated injury of the coronary vasculature.
\end{abstract}

\section{Introduction}

Heart transplantation is the most effective treatment of end-stage heart failure to prolong life. Modern immune-suppression regimens blunt alloreactive immune responses against the transplant, but are nevertheless associated with appreciable rates of early acute cellular rejection and late chronic cell- and antibody-dependent graft injury (1). Long-term heart allograft survival is primarily limited by chronic allograft rejection (2). Currently, no treatment exists for this occlusive chronic allograft vasculopathy (CAV) that is the lead cause of recipient death.

The transplant coronary artery endothelium is the principal target of the indolent immune response (3). Recent examination of failing heart grafts explanted for retransplantation of the recipient has identified that the entire vascular tree, from the coronary artery to the microvasculature, of the allograft is damaged (4). Cell-mediated injury is required to elicit vasculopathy $(5,6)$. Immune responses dominated by IFN- $\gamma$ production and mononuclear cell infiltration of the graft arteries have been demonstrated in mouse models (7-9) and among human heart transplants with

Conflict of interest: CF, JCV, and GYO have an intellectual property claim pending on the apelin-17 analoG used in these experiments (N-MeLeu9-apelin-17; patent application no. PCT/IB2018/000837).

Copyright: 단 2020, American Society for Clinical Investigation.

Submitted: February 28, 2019; Accepted: September 18, 2019;

Published: November 18, 2019

Reference information: / Clin Invest. 2020;130(1):94-107.

https://doi.org/10.1172/JCI128469. vasculopathy (10), and mechanistically linked to the development of vasculopathy in humanized mouse models (11-13).

Injury to the arterial or microvascular endothelium elicits a repair response, but little is known regarding the mediators involved in repair of the damaged mature vasculature. Proangiogenic mediators are better defined in development and tumor neo-angiogenesis (14). Among these, vascular endothelial growth factor (VEGF) is a dominant growth cue, but angiogenesis is modified by a variety of ligands for $G$ protein-coupled receptors. These cues elicit differentiation of "tip" endothelial cells (ECs) leading an angiogenic sprout to acquire motility, and elaborate soluble and matrix-associated molecules to crosstalk with adjacent trailing ECs and neighboring smooth muscle cells $(15,16)$.

Apelin is an EC-derived peptide agonist for the apelin G protein-coupled receptor $(15,17)$. Apelin is best characterized for its effect as a potent inotropic agent for cardiac myocytes $(18,19)$. However, apelin is induced in the tip EC directly by tissue hypoxia and indirectly by VEGF, and signals to trailing stalk ECs that express the apelin receptor $(20,21)$. Apelin loss in the developing embryo is associated with subtle defects in vascularization $(18,22)$. In the adult, apelin is required for tip EC sprouting during regenerative angiogenesis after tailfin amputation in zebrafish (21), and plays a role in myocardial remodeling after infarction (23).

Here we tested the hypothesis that the developmental angiogenesis program contributes to vascular endothelial repair of the established adult coronary circulation under chronic immune injury. We define expression of characteristic EC-enriched tip cell transcripts to mark vascular repair in murine and human arteries 
and microvessels after heart transplantation. Of these, we focused on apelin, since apelin signaling to vascular ECs might cue repair and be amenable to therapeutic intervention. We find that apelin expression by the graft vasculature is critical to mediate vascular repair, and defend the graft against immune cell invasion. An apelin receptor agonist mitigates both immune cell infiltration and maladaptive vascular repair.

\section{Results}

Endothelial tip genes are expressed during immune injury. First, we sought to determine whether injury of the established mammalian vasculature is accompanied by reparative endothelial differentiation in vivo. Initially, heart transplantation is associated with reperfusion injury to the graft vasculature. Later, the vascular endothelium of the male heart graft coronary artery and microvessels is known to be targeted by the $\mathrm{H}-\mathrm{Y}$ minor histocompatibility antigen-directed alloimmune response in female mice $(24,25)$. To confirm injury, we characterized endothelial microvascular density in the myocardium 2 weeks after heterotopic heart transplantation of a male donor heart to a female major histocompatibility complex-matched recipient mouse. We observed about $45 \%$ loss of microvascular profiles in sections immunostained for endothelial CD31 or cadherin 5 among hearts transplanted to female or male recipients versus normal heart tissue, consistent with ischemia/ reperfusion injury-induced loss (Figure 1A). We evaluated gaps in the continuity of the endothelium in coronary artery cross-section profiles of grafts after transplantation. Focal EC loss was seen in grafts of female, but not male, heart recipients (Figure 1B, left, and Supplemental Figure 1A; supplemental material available online with this article; https://doi.org/10.1172/JCI128469DS1). Similarly, we observed an increase in cleaved caspase- 3 costaining of $\mathrm{CD} 31^{+}$ECs among hearts transplanted to female recipients, consistent with apoptotic stress of the endothelium (Figure 1B, right, and Supplemental Figure 1B). Fibrin immunostaining was associated with the injured arterial and microvascular endothelium of hearts transplanted to female recipients (Supplemental Figure 1C). These data indicate that the loss of microvessel density at 2 weeks after transplantation is largely attributable to earlier reperfusion injury, and suggest additional active vascular injury in the allograft, but not syngeneic graft.

Angiogenesis in the developing vasculature, cancer neo-angiogenesis, and sprouting from endothelial spheroids cultured in vitro are associated with characteristic genes expressed by the lead tip EC (15, 26-29). We hypothesized that tip cell genes are similarly expressed during repair of the endothelium of the established vasculature. Among genes known to be upregulated by the tip cell during angiogenesis, we selected Apln, Egfl7, Esm1, and Pdgfb, as readouts with endothelial-selective expression. We examined tip gene expression in epicardial coronary arteries microdissected from the myocardium of the heart transplant (Figure 1C). Since only small amounts of RNA were isolated from the epicardial left coronary arteries, specimens were pooled in pairs, then analyzed for expression of the selected genes. We observed that each tip cell gene was markedly upregulated at 2 weeks after transplantation in the allogeneic male hearts (Figure 1C and Supplemental Figure 2), whereas little change in expression was seen with the constitutively expressed, endothelial-specific gene Pecam (CD31). Moreover, high tip cell gene expression persisted at 6 weeks after transplantation. In contrast, tip cell gene expression among male-to-male transplanted hearts at 2 and 6 weeks after transplantation was similar to that in freshly isolated native heart tissue, consistent with resolution of reperfusion injury-associated repair that occurred at the time of transplant. Remarkably, we observed a parallel pattern of expression of the tip genes among coronary artery and the heart microvascular ECs in the myocardium (Figure 1, C and D).

To confirm protein expression, we examined deposition of the tip cell matrix protein ESM1 in the heart by immunohistochemistry. As shown in Figure 1, E and F, focal deposits of ESM1 were found in the myocardium associated with CD31+ microvessels, and in the wall of the expanded arterial intima, in the allogeneic, but not syngeneic, heart transplants. Similarly, allografts upregulated expression of EGFL7 and apelin in the arterial endothelium (Supplemental Figure 2 and Supplemental Figure 3, A and $\mathrm{B}$ ). These findings are consistent with resolution of an early wave of repair in the male-to-male heart transplants, and indicate vascular repair in response to active injury from the alloimmune response in the male-to-female heart transplants. Notably, the repair genes were expressed in the isolated artery, indicating that arterial repair is associated with a repair program similar to that of the heart microvasculature.

Apelin loss decreases endothelial repair gene expression. Loss of apelin receptor signaling is associated with defects in vascular development $(18,22)$; hence we hypothesized that apelin cues vascular repair in the allograft. To test this, we transplanted hearts from apelin-deficient male donors to major histocompatibility complex-matched, apelin-WT female recipient mice.

We examined the effect of apelin loss on endothelial reparative gene expression. Apelin expression in the apelin-deficient hearts was undetectable by quantitative reverse transcriptase PCR (qRT-PCR), indicating that apelin is endogenous to the graft vasculature and is not supplied by circulating donor progenitor cells or infiltrating leukocytes (Figure 1, C and D). We found that apelin loss abolished the induction of endothelial tip cell gene expression in the isolated artery and myocardium (Figure 1, C and D). Similarly, we found that deposition of ESM1 and EGFL7 protein markedly decreased in the perivascular matrix of the apelin-deficient hearts (Figure 1, E and F, and Supplemental Figure 2). In contrast, expression of Vegfa, produced by parenchymal cells and leukocytes infiltrating the graft coronary artery and myocardium, remained elevated, consistent with a proangiogenic tissue microenvironment (Supplemental Figure 4).

Apelin loss exacerbates vasculopathy. We found that intimal expansion and occlusion of the arterial lumen was more pronounced among apelin-deficient versus WT littermate heart allografts (Figure 2, A and B). Apelin loss in the allograft further decreased the microvessel density versus transplanted male control hearts (Figure 1A, Figure 2C, and Supplemental Figure 5). In contrast, no effect of apelin loss was seen on the normal arterial histology after reperfusion injury among the syngeneic grafts (Figure 2B).

Apelin receptor agonist promotes endothelial repair in vitro. We studied the effect of apelin receptor stimulation on endothelial repair in vitro. Functionally, knockdown of apelin by siRNA treatment of ECs decreased monolayer repair of a scratch wound in response to VEGF (Figure 2D). Further, apelin receptor agonist 

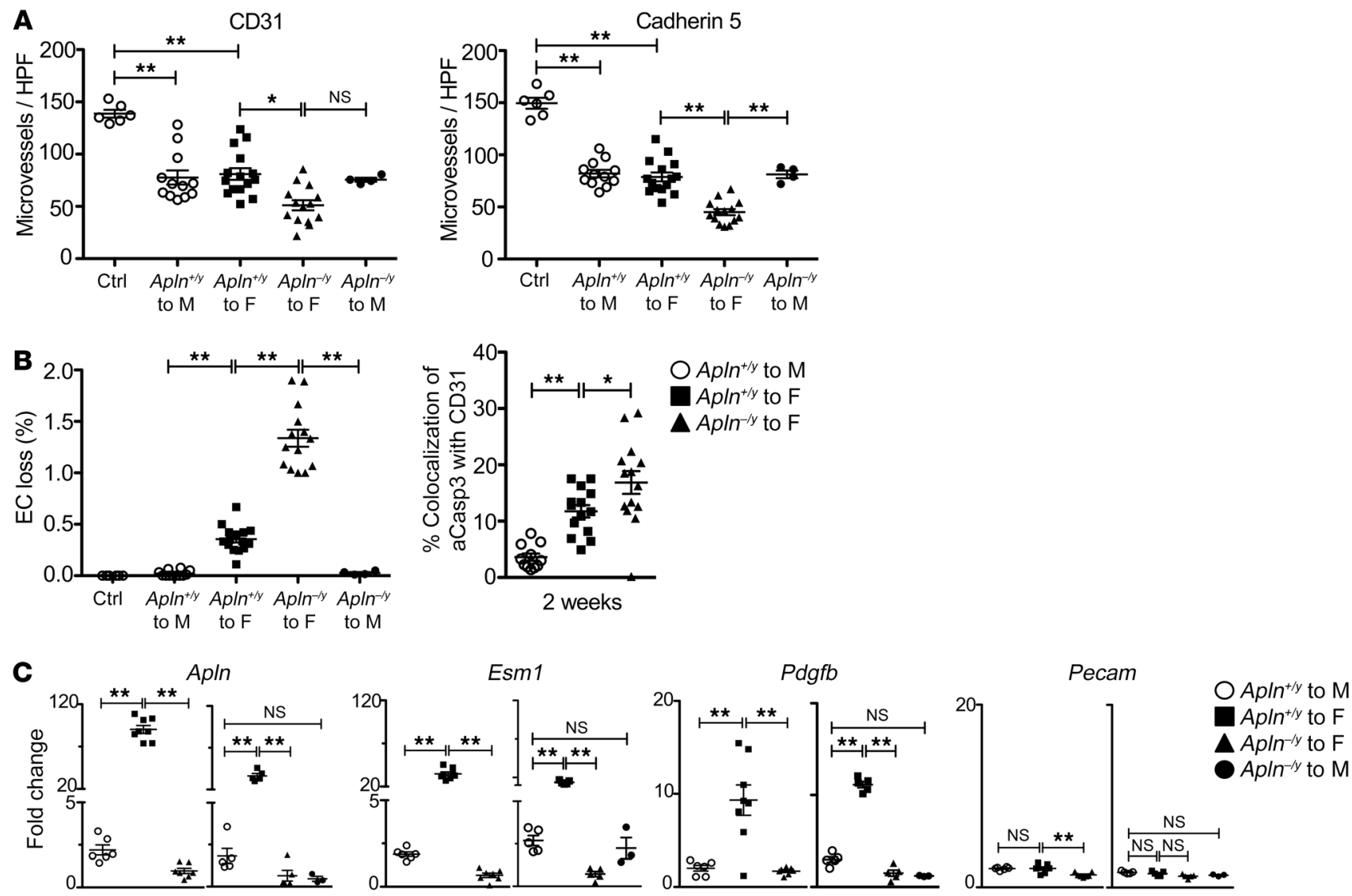

D
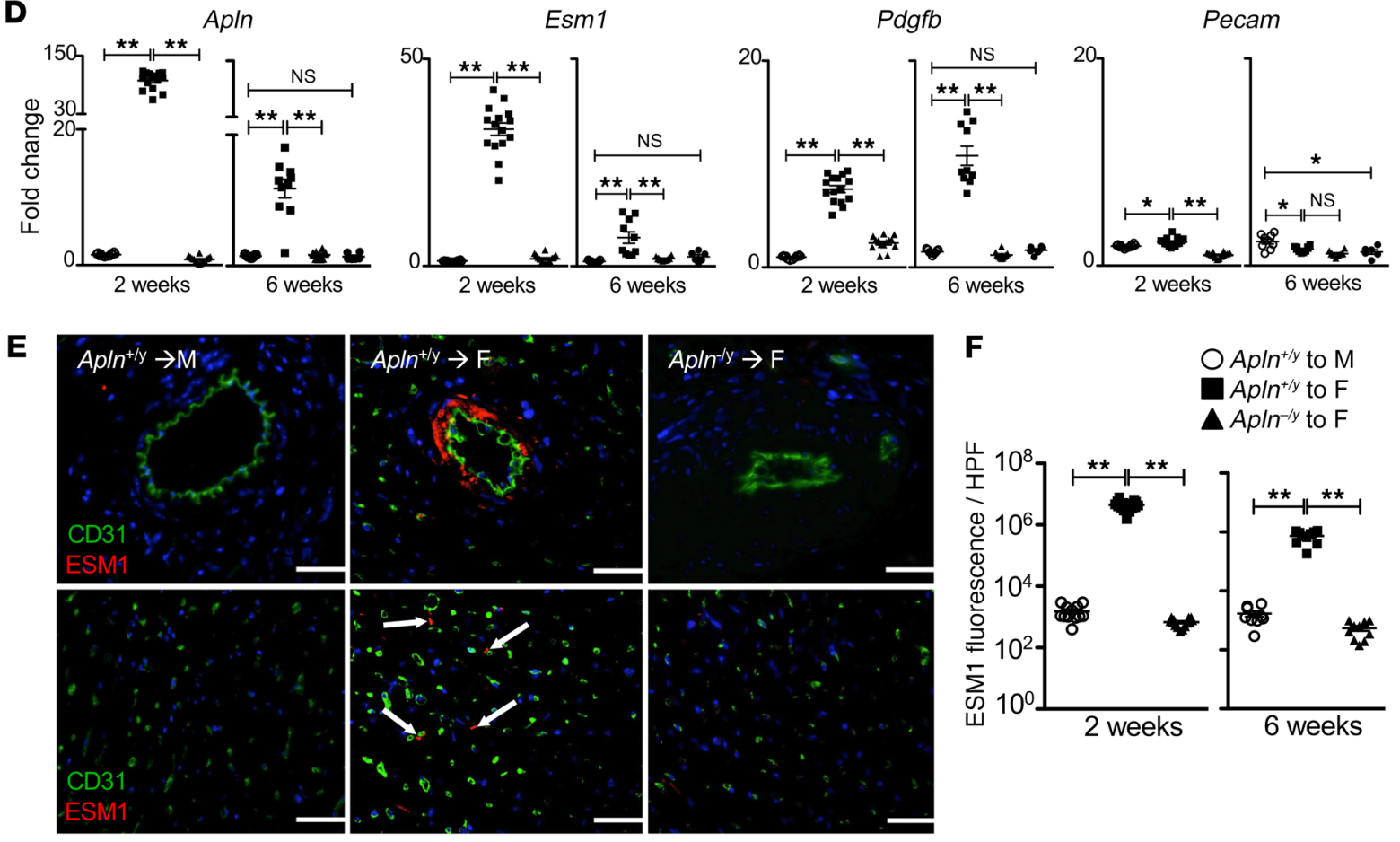
Figure 1. Post-transplant vascular injury is associated with endothelial repair gene expression. (A) Hearts recovered 2 weeks after transplantation were immunostained for the EC marker CD31 or cadherin 5. Myocardial microvessel density was quantitated (CD31+ [PECAM], left; cadherin $5^{+}$, right). Apl $n^{+/ y}$ to male recipients ( $n=12$ biological replicates) experienced reperfusion injury alone; $A p l n^{+/ y}(n=15)$ and $A p / n^{-/ y}(n=14)$ to $A p / n^{+/+}$ female recipients experienced reperfusion and chronic alloimmune injury. HPF, high-power field. (B) Gaps in the arterial endothelium in cross section (left) and the fraction of cleaved caspase- $3^{+}$(aCasp) immunostaining among the $\mathrm{CD} 1^{+}$arterial endothelium (right) were quantitated among the samples from $\mathbf{A}$. Endothelial repair gene expression among transplanted hearts was determined by qRT-PCR, and expressed relative to nontransplanted control hearts. (C) Gene expression among microdissected coronary arteries at 2 or 6 weeks after transplantation. Samples were pooled in pairs for analysis (at 2 weeks: $A p / n^{+/ y}$ to male recipients, $n=6$ pairs; $A p / n^{+/ y}$, $n=8$, and $A p l n^{-1 /}, n=7$, to $A p / n^{+/+}$female recipients; at 6 weeks: $A p / n^{+/ y}$ to male recipients, $n=5$ pairs; $A p l n^{+/ y}, n=5$, and $A p / n^{-/ y}, n=5$, to $A p / n^{+/+}$ female recipients). (D) Cene expression among myocardium samples after transplantation (at 2 weeks: $A p / n^{+/ y}$ to male recipients, $n=12$ biological replicates; $A p / n^{+/ y}, n=15$, and $A p / n^{-/ y}, n=14$, to $A p / n^{+/+}$female recipients; at 6 weeks: $A p / n^{+/ y}$ to male recipients, $n=9$ biological replicates; $A p / n^{+/ y}$, $n=10$, and $A p / n^{-/ y}, n=10$, to $A p / n^{+/+}$female recipients). (E) Hearts recovered 2 weeks after transplantation were immunostained for endothelial CD31 (green) and ESM1 (red, arrows). Medium-sized to larger arterial cross sections are represented in the top panels, whereas myocardial microvessels are in the bottom panels. Scale bars: $50 \mu \mathrm{m}$. (F) ESM1 immunofluorescence quantitation among heart transplants in $\mathbf{E} ; A p / n^{+/ y}$ to male recipients ( $n=12$ biological replicates), $A p / n^{+/ y}(n=15)$ and $A p / n^{-/ y}(n=14)$ to $A p / n^{+/+}$ female recipients. Mean $\pm \mathrm{SEM}$; ${ }^{*} P<0.05,{ }^{* *} P<0.01$, by 1 -way ANOVA with Bonferroni's post hoc test.

treatment using a proteinase-resistant apelin-17 analog (Supplemental Figure 6A and ref.30) augmented VEGF-stimulated scratch wound repair (Figure 2D and Supplemental Figure 6B). Scratch wound repair in vitro was associated with induction of ESM1, which was abolished by RNA interference-mediated knockdown of APLN (Figure 2, E and F). Moreover, sprouting and tip cell gene expression is induced by the apelin receptor agonist in $3 \mathrm{D}$ endothelial spheroid cultures in vitro (Supplemental Figure 6, C and D). These findings indicate that vascular endothelial reparative differentiation, reflected by induced expression of apelin and ESM1, directly mitigates vascular injury. Further, traditional proangiogenic cues such as VEGF are insufficient to optimally mediate repair.

Endothelial repair genes are expressed in human heart transplants. Next, we examined reparative endothelial gene expression in human heart transplants. We studied coronary artery and endomyocardial samples of end-stage human hearts with advanced transplant vasculopathy, explanted during retransplantation of the recipient (Figure 3A). Unutilized donor hearts were used as a reference sample. Features of atherosclerosis were evident in both sets of human coronary arteries, but the transplanted vessels had additional features of chronic allograft vasculopathy (CAV) (10), and in some cases prominent calcification of the expanded intima. A small intramyocardial artery in the reference sample shown in Figure $3 \mathrm{~A}$ has a patent lumen, and a single-cell intimal layer. In contrast, the intramyocardial artery in the explanted heart graft features marked lumenal narrowing by intimal myofibroblast proliferation, matrix accumulation, and chronic inflammation. Epicardial arteries from the explant show similar prominent intimal myofibroblast proliferation. In contrast, the reference heart artery shows established intimal fibrosis with cholesterol deposits. The intimal expansion among the explant arteries was marked (Figure 3B), consistent with advanced disease. The explant arteries heterogeneously expressed the repair genes (Figure 3C). We found an increase in APLN expression among the explant coronaries, and a trend for ESM1 induction, compared with the reference arteries. Apelin expression was detected by immunohistochemistry colocalized with endothelial CD34 in the explant arteries (Supplemental Figure 3C). The allograft samples with the most extensive intimal calcification had the lowest expression of the EC repair genes.

We also examined tip EC gene expression among the explant myocardium samples. We found upregulation of APLN, ESM1, and $P D G F B$ in comparison with the reference myocardium (Figure 3D). The magnitude of the reparative gene expression was similar to that of expression in the peri-infarct zone after myocardial infarction (data not shown).

Further, we characterized APLN, ESM1, and PDGFB expression in endomyocardial biopsies of the interventricular septum of functioning heart transplants, obtained at a mean of 3 (range 0.3-7) years after transplantation. We identified 9 recipients with transplant vasculopathy defined clinically by intravascular ultrasound and/or coronary angiography, and 5 recipients without vasculopathy. We compared gene expression among heart transplants with CAV, no CAV, and the nontransplanted donor hearts as the reference (Figure 3E). We observed elevated expression of the endothelial repair genes among the post-transplant samples with vasculopathy. Together, these data indicate that the endothelial repair gene program in the heart is conserved among mice and humans.

Apelin loss promotes inflammation. We examined the alloimmune response to the apelin-deficient graft. As reported, the male heart allograft is infiltrated by lymphocytes and monocytes at day 14 after transplantation, i.e., cellular rejection without an alloantibody response (31). We observed an increase in the number of lymphocytes and monocytes/macrophages at both 2 and 6 weeks after transplantation in the apelin-deficient grafts (Figure 4, A and B, and Supplemental Figure 7). There was little qualitative difference in the relative fractions of $\mathrm{CD}^{+}$versus $\mathrm{CD} 8^{+}$lymphocytes, $\mathrm{F} 4 / 80^{+}$macrophages, or M1 versus M2 monocytes, determined by marker immunohistochemical staining, in the graft among the apelin-deficient versus WT hearts. However, M1 monocyte infiltration of the arterial intima was more prominent among apelin-deficient hearts at 2 weeks (Figure 4B). Further, we found that IFN- $\gamma$-dependent CXCL11 transcript expression was increased early in the rejection response among apelin-deficient hearts in both the artery and myocardium compartments (Figure 4C). Similarly, IFN- $\gamma$ was markedly increased in the 2-week apelindeficient myocardium samples (Figure 4D). IFN- $\gamma$ was comparable among apelin-deficient and WT grafts at the later time point, but increased CXCL11 expression persisted among the apelin-deficient hearts. The proinflammatory cytokine TNF- $\alpha$ was modestly higher among apelin-deficient hearts (Figure 4D).

Since lymphocyte-generated IFN- $\gamma$ has been linked to CAV in human clinical material, we further characterized the frequency of alloreactive lymphocytes between recipients of apelindeficient and WT grafts. The spleen is the primary site of alloantigen presentation after murine heart transplantation (32). Therefore, we harvested splenic lymphocytes 2 weeks after 
A

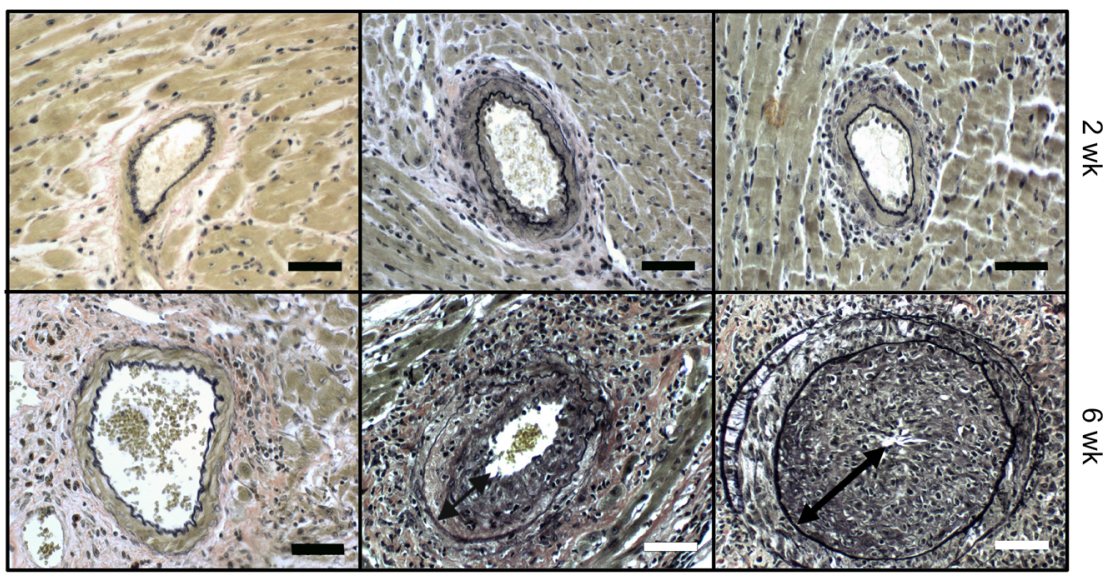

$A p / n^{+/ y}$ to $\mathrm{M}$
$A p / n^{+/ y}$ to $\mathrm{F}$

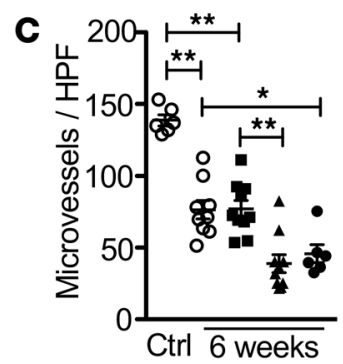

$A p / n^{-/ y}$ to $\mathrm{F}$

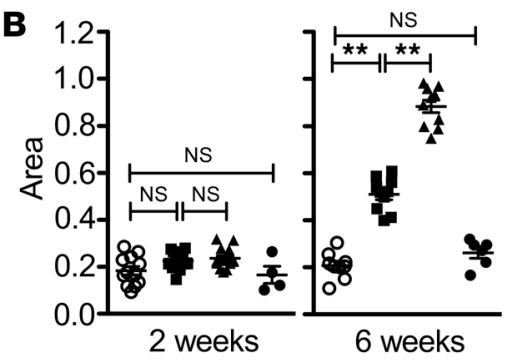

O Apl $n^{+/ y}$ to $\mathrm{M}$ Aplnt/y to $\mathrm{F}$ $\Delta A^{-1 y} I^{-1 y}$ to $\mathrm{F}$ Apln ${ }^{-1 y}$ to $\mathrm{M}$
D

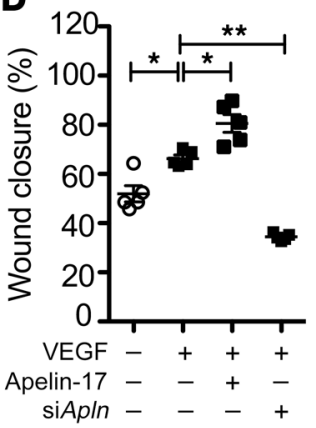

$\mathbf{E}$

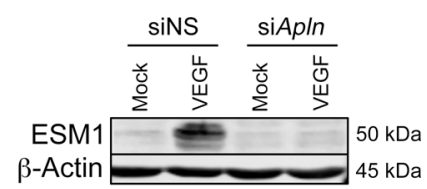

F

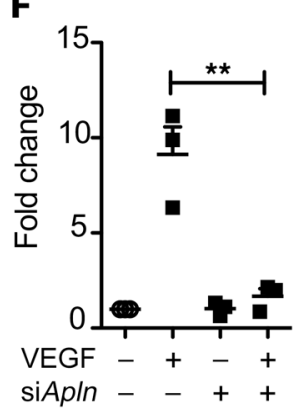

Figure 2. Apelin loss exacerbates arterial vasculopathy and blunts endothelial repair. (A) Photomicrographs of coronary arteries from mouse heart transplants, with van Gieson stain to highlight the internal elastic lamina in black. Syngeneic male-to-male transplants have a single-cell layer of intima. Progressive expansion of the intima is evident from 2 weeks (top panels) to 6 weeks (bottom panels) after transplantation in allogeneic male to apelin WT female transplants, which is more marked among apelin-deficient male donor hearts. Scale bars: 50 $\mu \mathrm{m}$. (B) Quantitation of intima area as a fraction of the area within the internal elastic lamina at 2 weeks and 6 weeks after transplantation among grafts as in Figure 1. (C) Microvessel density among freshly harvested donor reference hearts versus WT or apelin-deficient hearts from B recovered 6 weeks after transplantation. Mean $\pm \mathrm{SEM} ;{ }^{*} P<0.05,{ }^{*} P<0.01$ by 1 -way ANOVA with Bonferroni's post hoc test. (D) ECs were transfected with scrambled or apelin siRNA, then plated at confluence. The monolayers were injured with a scratch wound, then stimulated with VEGF (50 $\mathrm{ng} / \mathrm{mL})$ or apelin-17 analog (1 $\mu \mathrm{M}$ ) as indicated ( $n=5$ biological replicates in independent experiments). Mean $\pm \mathrm{SEM}$; ${ }^{*} P<$ $0.05,{ }^{* *} P<0.01$ by 1 -way ANOVA with Bonferroni's post hoc test. (E) ECs were transfected with scrambled or apelin siRNA, then plated at confluence. The monolayers were injured with multiple scratch wounds, then stimulated with VEGF $(50 \mathrm{ng} / \mathrm{mL}$ ) or mock-treated. Western immunoblot of EC lysates for ESM1. Representative of 3 biological replicates. (F) Quantitation of data in $\mathbf{E}$ ( $n=3$ biological replicates). Mean \pm SEM; ${ }^{* *} P<0.01$ by 1 -way ANOVA with Bonferroni's post hoc test. transplantation, and quantitated the frequency of alloresponsive lymphocytes among the recipient mice using $\mathrm{H}-\mathrm{Y}$ antigen $\mathrm{I}-\mathrm{A}^{\mathrm{b}}$ tetramers and flow cytometry. We observed no increase in the frequency of total, CD $44^{\text {hi }}$ memory, or IFN- $\gamma$-positive, alloreactive lymphocytes among the apelin-deficient versus WT recipients (Figure 4E). This strongly argues that apelin loss does not render the graft more immunogenic, or promote expansion of the alloreactive lymphocyte population.

We reasoned that apelin loss may alter leukocyte trafficking to the graft through autocrine signals among ECs. First, we reduced apelin receptor expression among ECs in vitro using RNA interference (Figure 5A). Apelin is known to signal to vascular ECs to elicit AKT and endothelial nitric oxide synthase (eNOS) activity. We confirmed that an apelin-17 analog acts as a selective apelin receptor agonist (30). Apelin-17-stimulated AKT and eNOS phosphorylation required endothelial apelin receptor expression (Figure 5, B and C).

To test the hypothesis that apelin receptor stimulation may repel leukocyte entry to the heart, we stimulated EC monolayers with TNF- $\alpha$ for 18 hours to simulate inflamed endothelium, then evaluated adhesion of primary human monocytes to the monolayer. We found that monocyte adhesion to an EC monolayer in vitro was decreased with apelin receptor stimulation of the monocyte/EC coculture, in an eNOS-dependent fashion (Figure 5D and Supplemental Figure 8).

Apelin-17 treatment blocks progression of transplant vasculopathy. To determine whether an apelin receptor agonist treatment could modify graft coronary arterial injury, we treated recipient mice daily with the synthetic apelin receptor agonist apelin-17 peptide, which is resistant to degradation by endogenous plasma proteinases (30). Treatment was begun at 2 weeks after transplantation to model the clinical scenario of treatment of early, established injury. Hearts were harvested at 6 weeks after transplantation, and the extent of graft vascular injury was evaluated. We found that the graft coronary artery intimal expansion was markedly decreased in the apelin receptor agonist-treated versus saline-treated control grafts (Figure 6, A and B), and we observed a marked reduction in arterial gaps and cleaved caspase-3 staining of the endothelium among the treated hearts (Supplemental Figure 9). Further, allograft microvessel density was increased among the apelin-17- 
A

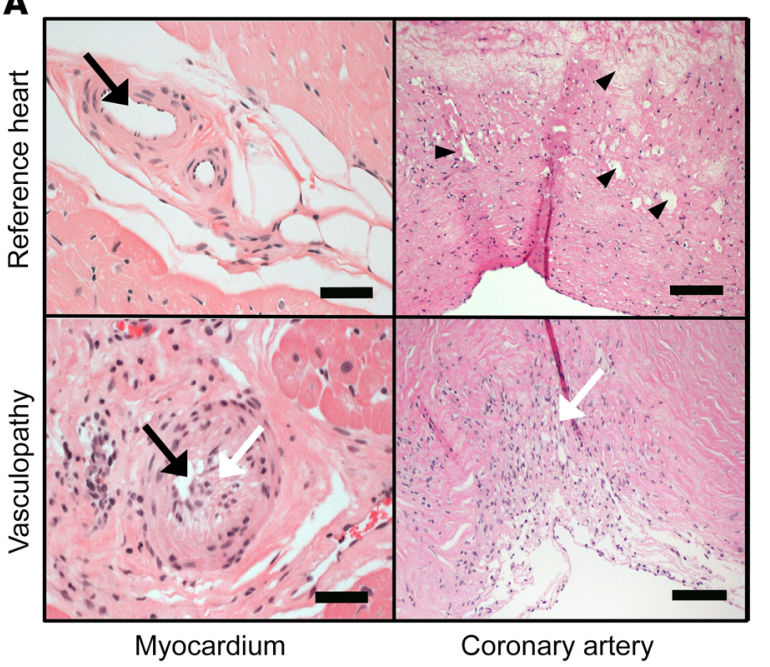

B

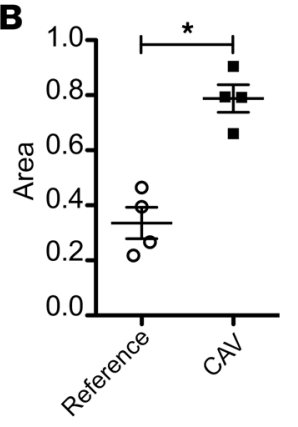

C
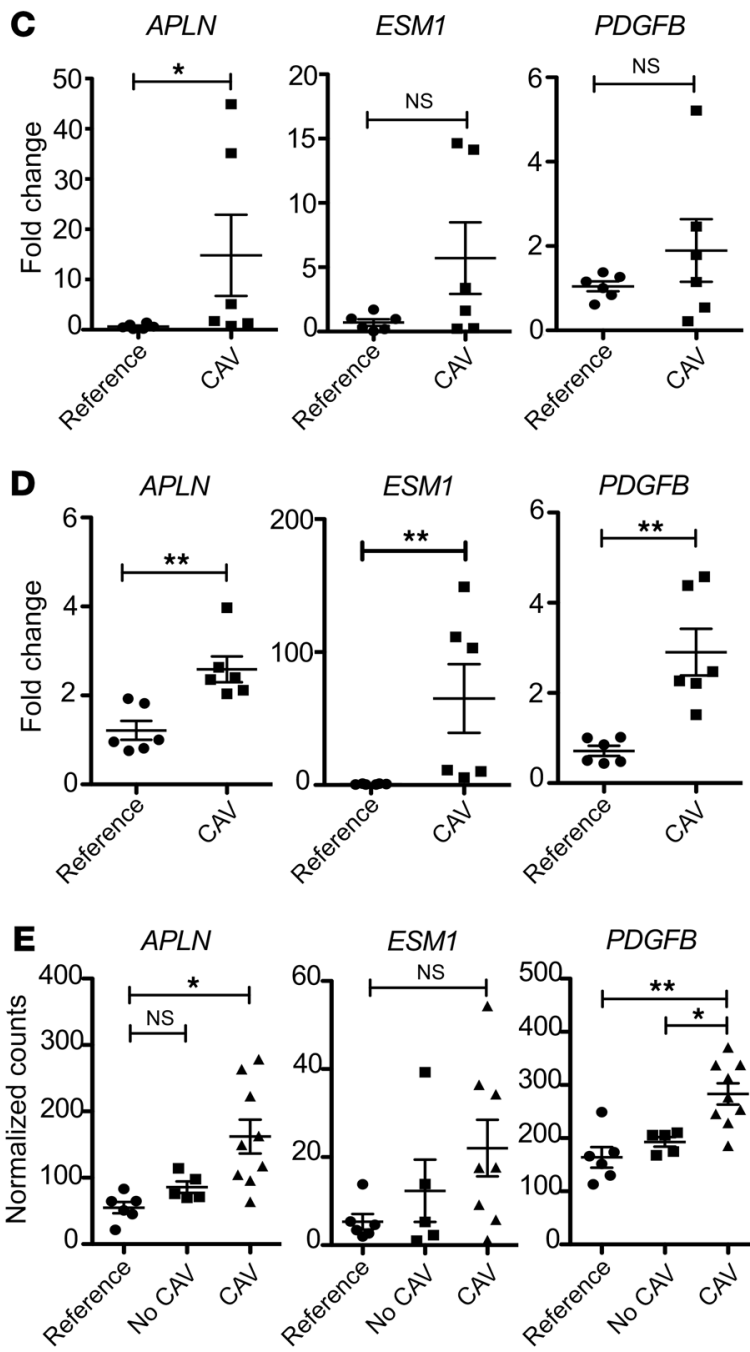

PDGFB

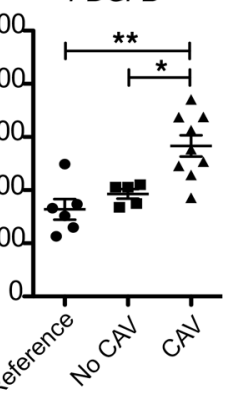

Figure 3. Endothelial repair genes are expressed in injured human hearts. (A) Histology of coronary artery and myocardium of human hearts, H\&E stain. Top panels show an unutilized donor reference heart. Bottom panels show a transplant heart explant with arterial CAV, recovered at the time of recipient retransplantation. The arterial lumen (black arrows) is occluded in the explant, with prominent myofibroblasts (white arrows) and matrix in the explant intima. The reference arteries show atherosclerotic cholesterol accumulation (arrowheads). Scale bars: $50 \mu \mathrm{m}$ (left panels), $200 \mu \mathrm{m}$ (right panels). (B) Quantitation of the intima area as a fraction of the area within the internal elastic lamina of epicardial coronary arteries among unutilized donor and explant hearts ( $n=4$ biological replicates). (C) Expression of endothelial repair genes in coronary artery of reference or explant hearts ( $n=6$ biological replicates). (D) Expression of endothelial repair genes in myocardium among reference ( $n=6$ biological replicates) or explant allograft hearts with vasculopathy $(n=6)$. (E) Expression of endothelial repair genes in myocardium among endomyocardial biopsy specimens of reference ( $n=6$ biological replicates) or transplant hearts with $(n=9)$ or without $(n=5)$ arterial CAV. Mean \pm SEM; ${ }^{*} P<0.05,{ }^{* *} P<0.01$ by 1-way ANOVA with Bonferroni's post hoc test.

treated animals (Figure 6, A and C). After 4 weeks of treatment, native Apln expression in the graft was suppressed in the isolated coronary artery (Figure 6F) and myocardium compartments (Figure 6H). Similarly, EC repair biomarker (Esm1, Pdgfb) and Vegfa expression was normalized versus untreated grafts in both isolated coronary arteries and myocardium.

We examined the effect of the apelin agonist treatment on leukocyte infiltration in the allograft. We found a markedly decreased number of $\mathrm{T}$ cells and monocytes infiltrating the apelin-17-treated allografts (Figure 6, A and D). In line with this observation, the allograft expression of IFN- $\gamma$ and that of TNF- $\alpha$ were both decreased versus the saline-treated hearts in both coronary artery and myocardium compartments (Figure 6, G and I, respectively). These data indicate that apelin receptor agonist treatment decreases graft immune cell infiltration with an accompanying decrease in proinflammatory cytokine generation.

We tested the hypothesis that apelin-17-stimulated nitric oxide was required to protect the graft from immune cell infiltration. L-NAME was administered with apelin-17 (33), and graft survival was evaluated. Inhibition of nitric oxide synthases reversed the effect of apelin-17 to reduce inflammation (Figure 6D), and promoted graft loss (Figure 6E). This was associated with coronary vasculopathy (Figure 6, B and C). Our findings show that apelin receptor agonist treatment both decreases the immune injury burden on the graft vasculature and promotes vascular repair resulting in normalization of endothelial repair gene expression. Conversely, inhibition of nitric oxide generation facilitates rejection and vascular injury despite apelin-17 treatment. 

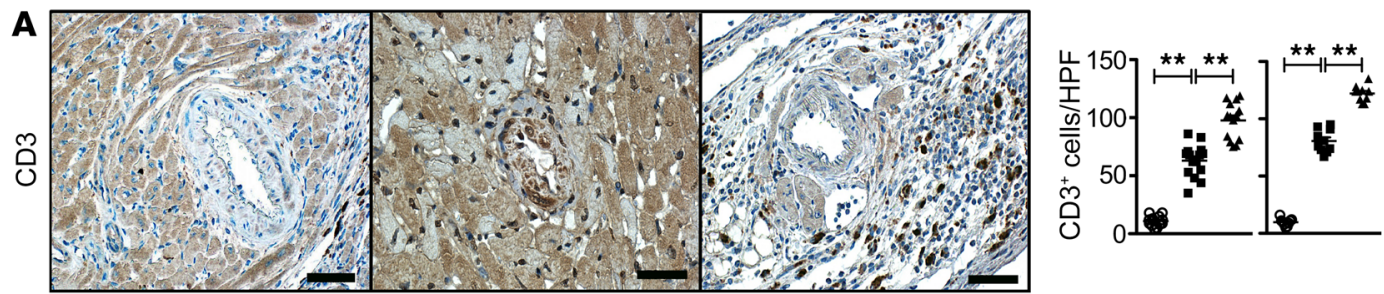

O Apln $n^{+/ y}$ to $\mathrm{M}$

Apln $n^{+/ y}$ to $\mathrm{F}$

$\Delta$ Apln ${ }^{-1 y}$ to $\mathrm{F}$
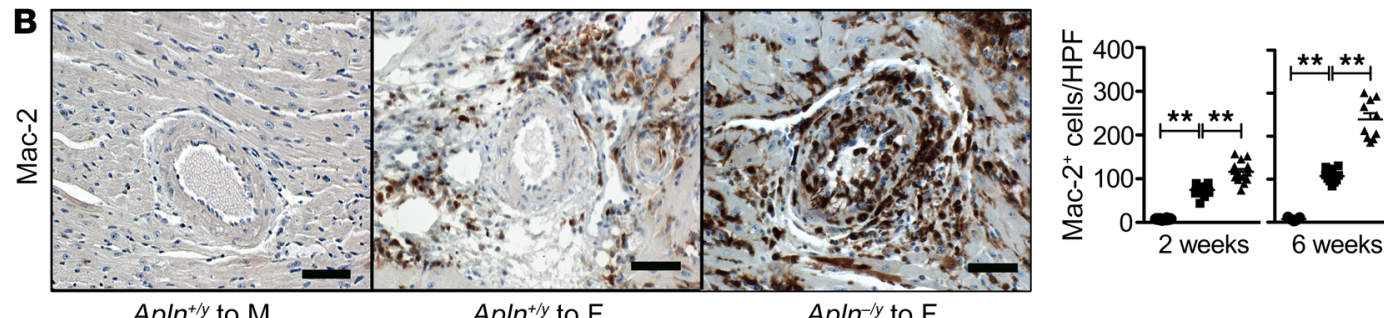

Ap/n $n^{+/ y}$ to $\mathrm{M}$

$A p / n^{+/ y}$ to $\mathrm{F}$

$A p / n^{-1 y}$ to $\mathrm{F}$

C

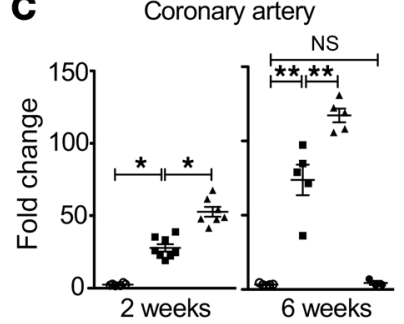

Myocardium

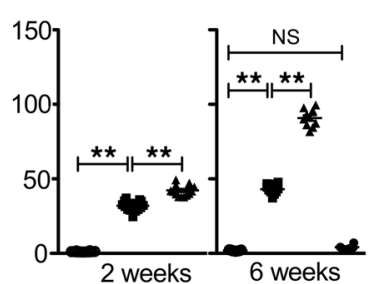

D
Tnfa

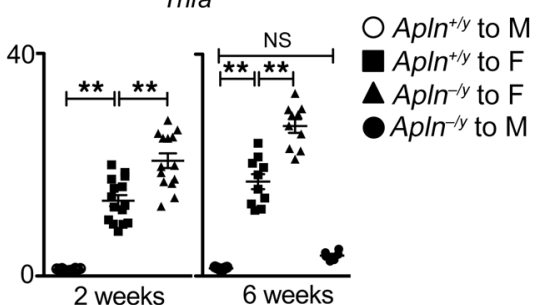

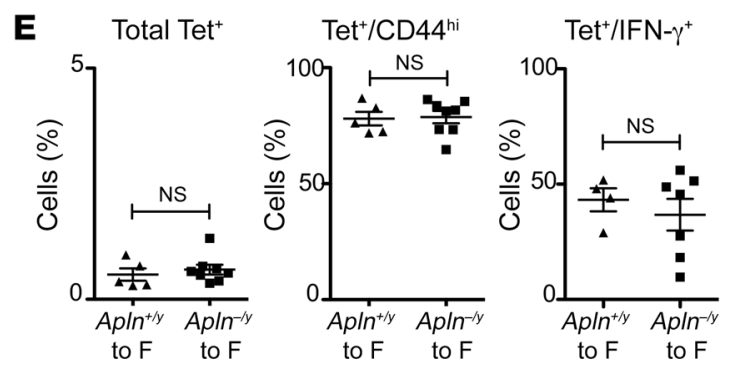

Figure 4. Apelin loss increases heart inflammation after transplantation. Hearts were recovered after transplantation. (A and B) Immunostains for CD3, a lymphocyte marker (left panel), with quantitation at 2 weeks and 6 weeks after transplantation among grafts as in Figure 1 (A); and for Mac-2, a monocyte/macrophage marker (left panel), with quantitation at 2 weeks and 6 weeks after transplantation among grafts as in Figure 1 (B). Mean \pm SEM; ${ }^{* *} P$ $<0.01$ by 1-way ANOVA with Bonferroni's post hoc test. Scale bars: $50 \mu \mathrm{m}$. (C and $\mathbf{D})$ Expression of proinflammatory cytokines among heart transplants. (C) CXCL11 expression among microdissected coronary arteries and myocardium at 2 and 6 weeks after transplantation among grafts as in Figure 1. Mean $\pm \mathrm{SEM} ;{ }^{*} P<0.05,{ }^{* *} P<0.01$ by 1 -way ANOVA with Bonferroni's post hoc test. (D) Myocardial cytokine expression at 2 and 6 weeks after transplantation among grafts as in Figure 1. Mean $\pm \mathrm{SEM} ;{ }^{* *} P<0.01$ by 1 -way ANOVA with Bonferroni's post hoc test. (E) Recipient anti-male H-Y-alloreactive lymphocytes were isolated from the spleen at 2 weeks after transplantation, identified by staining with $\mathrm{H}-\mathrm{Y} \mathrm{I}-\mathrm{A}^{\mathrm{b}}$ tetramers, and characterized by dual staining with CD44 and intracellular IFN $-\gamma$. The data from individual mice are displayed. Mean $\pm \mathrm{SEM} ; P=\mathrm{NS}$ by Mann-Whitney test.

\section{Discussion}

In this study we sought to determine whether the embryonic vascular development program expressed by vascular ECs contributes to repair of the established vasculature in the adult. We focused on 3 biomarkers, apelin, ESM1, and PDGFB, selectively expressed in ECs, and well characterized in mouse developmental angiogenesis, to report on angiogenic "tip" cell differentiation in the heart. We found transient upregulation of the angiogenic biomarker panel in the myocardium after ischemia/reperfusion injury associated with isogeneic heart transplantation. However, transplanted hearts exposed to chronic alloimmune injury showed persistent expression of biomarker RNA and protein. Thus, despite similar density of ECs, the proangiogenic biomarkers reveal underlying endothelial repair among the allogeneic heart transplants. We found that this upregulated expression was conserved among samples of 2 independent groups of human transplanted hearts: those undergoing explant during recipient retransplantation for end-stage allograft vasculopathy, and those with developing vasculopathy. Moreover, the tip cell genes were induced in the periinfarct zone of human hearts after myocardial infarction. Thus the endothelial repair gene expression in response to immune injury is conserved in mice and humans.

Further, we examined the expression of the biomarkers in microdissected epicardial coronary arteries in the mouse heart transplants. This segment of the coronary vasculature is recognized to be prone to developing occlusive vasculopathy lesions in 
A
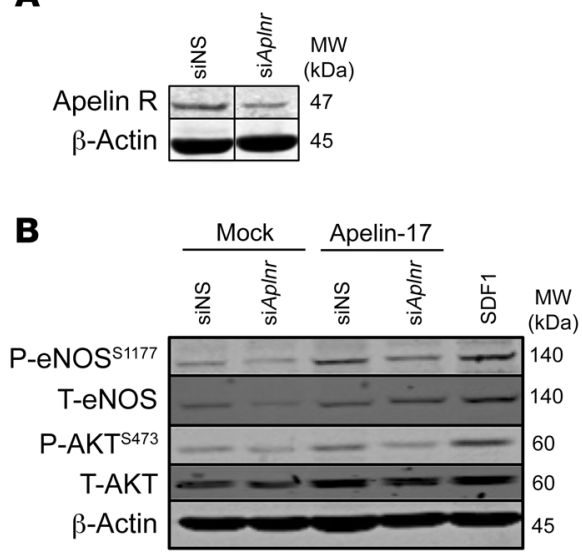

C

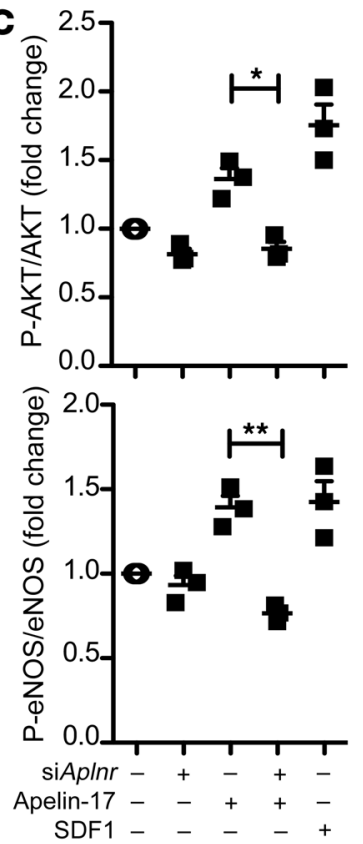

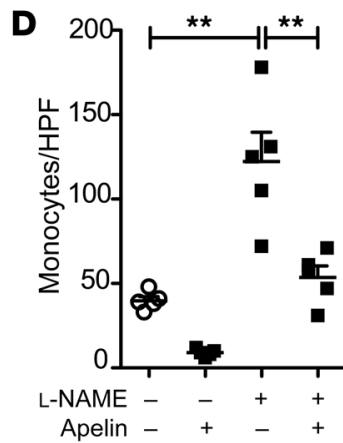

Figure 5. Apelin stimulates EC eNOS activation and inhibits monocyte adhesion. (A) Primary human ECs were transfected with siRNA against the apelin receptor, or scrambled siRNA, then lysed, and the apelin receptor was immunostained on Western blot. Representative of 4 biological replicates. (B) Apelin receptor stimulation of human ECs elicits phosphorylation of AKT and eNOS. (C) Quantitation of the data in B $(n=3$ biological replicates). Mean \pm SEM; ${ }^{*} P<0.05$, ${ }^{* *} P<0.01$ by ANOVA. (D) Human ECs were stimulated with TNF- $\alpha$ overnight, then cocultured with human monocytes. As indicated, the cocultures were mock-treated or treated with proteinase-resistant apelin-17 $(1 \mu \mathrm{M})$, DMSO, or the eNOS inhibitor L-NAME (100 $\mu \mathrm{M})$. The number of adherent monocytes was quantitated ( $n=5$ biological replicates). Mean \pm SEM; ${ }^{* *} P<0.01$ by 1-way ANOVA with Bonferroni's post hoc test.

humans. Notably, the biomarker panel expression was similarly regulated among paired coronary artery and myocardium samples in the mouse. This is consistent with the observation that the transplant vasculature in humans undergoes alloimmune injury along the length of the arterial tree, from artery to the microvasculature (4). Recent work identifies reparative endothelial progenitor cells embedded in the mouse aortic endothelium, but repair from mechanical injury involved EC dedifferentiation and proliferation (34). We confirmed that apelin expression in human heart explant coronary artery endothelium was induced versus unutilized donor arteries, but these specimens had advanced disease with heterogeneous expression of the biomarkers, and may not all reflect evolving lesions. Taken together, the findings suggest that biomarker interrogation of clinical endomyocardial samples reflects events in the arteries, but the features of the response may depend on the nature or duration of the injury.

Apelin, PDGFB, and ESM1 are each associated with vascular defects in knockout mice $(22,35,36)$, consistent with the notion that expression of these genes by the injured vascular endothelium participates in vascular repair. For example, loss of ESM1 reduces matrix-bound VEGF presentation to ECs and perturbs capillary outgrowth (36). In vitro, loss of apelin signaling reduces monolayer wound repair and tip cell differentiation, whereas apelin itself is able to cue differentiation in cultured human ECs. To directly address the hypothesis that apelin signaling within the injured tissue compartment, i.e., the heart allograft, cues vascular repair, we found that heart allografts from donor knockout mice lacking expression of apelin exhibited more areas of denuded endothelium and developed more aggressive arterial occlusion than hearts from WT littermate donors. Apelin-deficient allografts failed to induce expression of reparative tip cell markers in both the arterial and microvascular compartments. Microvascular density was decreased among the apelin-deficient allografts. VEGF appears insufficient to mediate vascular repair and induce expression of EC tip cell genes in the absence of apelin signals, since we find abundant expression of VEGFA among both apelin-sufficient and -knockout heart allografts. Optimal induction of reparative ECs in the adult requires coincident signaling by VEGF and apelin, and perhaps a series of supporting cues $(22,37,38)$.

Apelin loss increased, and apelin receptor agonist treatment suppressed, neointima expansion and vascular smooth muscle cell (VSMC) accumulation under chronic immune injury. This finding contrasts with neointimal formation in arterial ligation and atherosclerosis-prone mouse models, where apelin signaling directly to VSMCs is reported to drive myofibroblast migration and expansion $(39,40)$. Our observation that apelin loss is associated with reduced expression of PDGFB indicates that paracrine cues, intrinsic to the vascular wall, that drive VSMC movement and myofibroblast differentiation are blunted in the setting of apelin deficiency $(41,42)$. Conversely, EC-derived EGFL7 production, reported to inhibit VSMC migration (43), is also blocked in apelin-deficient hearts. However, the VSMC response to the intima microenvironment is affected by leukocyte-derived mediators that are also influenced by apelin.

We find that loss of apelin expression by the heart vasculature promotes inflammation. Infiltrating immune cells supply VSMCs with growth factors and cytokines such as IFN- $\gamma$ known to drive transplant vasculopathy $(7,11,13)$. Our investigation of alloim- 


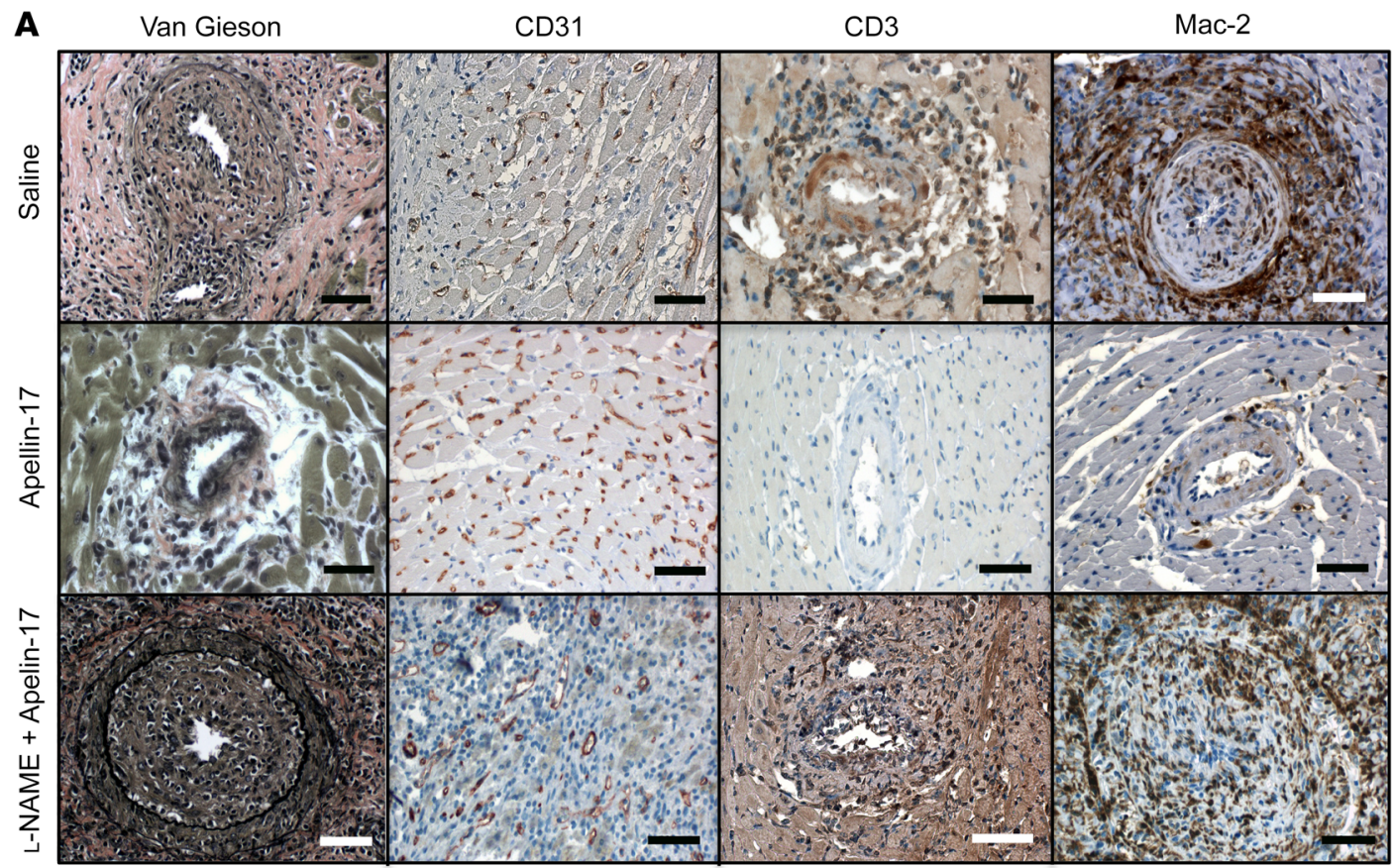

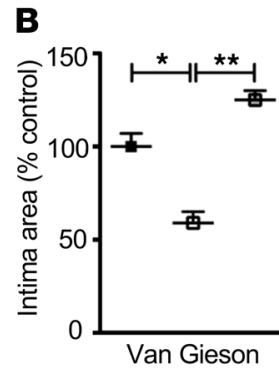

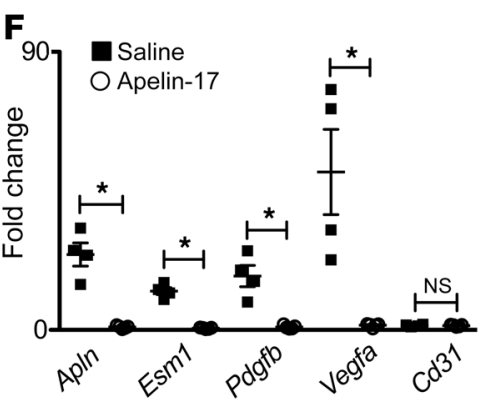

C

D

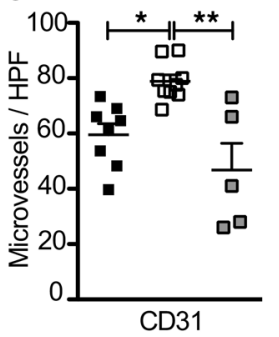

D

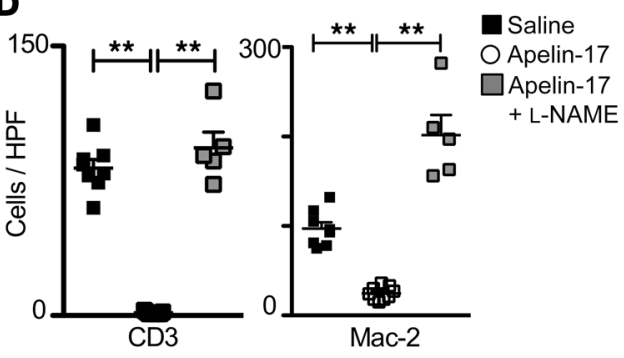

$\mathbf{E}$

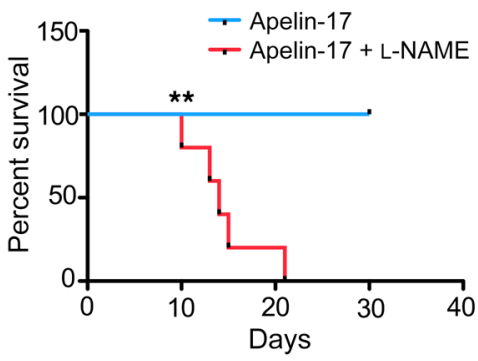

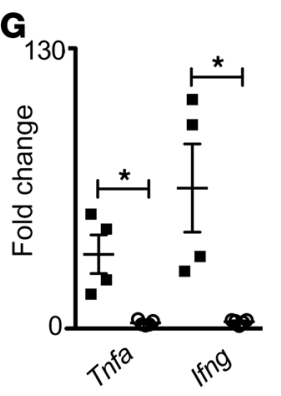
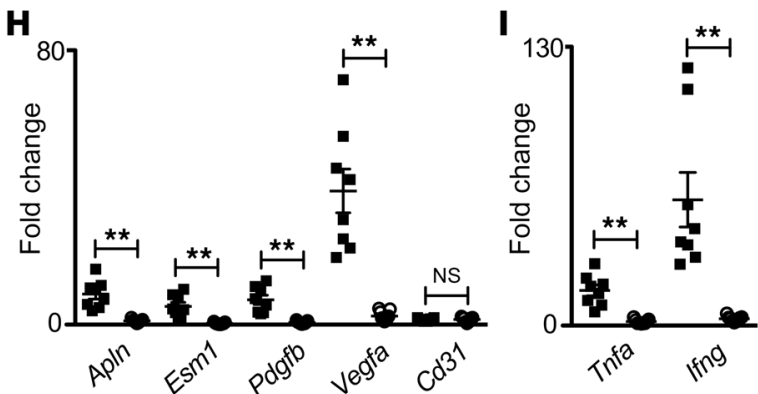

Figure 6. Apelin-17 analog treatment suppresses arterial vasculopathy and immune cell infiltration of heart transplants. Ap/n+/y male hearts were transplanted into WT female recipients. Two weeks after transplantation the recipient mice were treated daily with saline or apelin-17 analog or with apelin-17 analog plus L-NAME; then the heart allografts were recovered at the time the graft heartbeat stopped, or at 6 weeks after transplantation. (A) Photomicrographs of transplanted hearts stained with van Gieson, immunostained for CD3, Mac-2, or EC CD31. Scale bars: $50 \mu \mathrm{m}$. (B) Quantitation as in Figure 2 of the intima area of graft arteries in heart recipients treated with saline ( $n=8$ biological replicates), apelin-17 analog ( $n=9)$, or apelin-17 analog plus L-NAME $(n=5)$. (C) Quantitation of CD31+ microvessels in grafts of heart recipients treated with saline $(n=8$ biological replicates), apelin-17 analog $(n=9)$, or apelin-17 analog plus L-NAME $(n=5)$. (D) Quantitation of immune cell infiltration in myocardium of grafts from heart recipients as in C. Mean \pm SEM; ${ }^{*} P<0.05,{ }^{* *} P<0.01$ by 1 -way ANOVA with Bonferroni's post hoc test. (E) Survival of heart allografts in mice treated with apelin-17 analog without $(n=9)$ or with L-NAME ( $n=5$ biological replicates) starting on day 14 after transplantation. ${ }^{* *} P<0.01$ by log rank. (F and $\left.\mathbf{G}\right)$ Expression of endothelial repair $(\mathbf{F})$ and proinflammatory $(\mathbf{G})$ genes in microdissected coronary arteries from recipient mice treated with saline $(n=4$ pairs) or apelin-17 analog $(n=$ 5 pairs). Coronary artery data were analyzed by Mann-Whitney test. Mean $\pm \mathrm{SEM}$; ${ }^{*} P<0.05,{ }^{* *} P<0.02$. (H and $\mathbf{I}$ ) Expression of endothelial repair $(\mathbf{H})$ and proinflammatory (I) genes in myocardium of heart grafts from recipient mice treated with saline $(n=8)$ or apelin-17 analog $(n=9)$. Mean \pm SEM;

${ }^{* *} P<0.01$ by Student's $t$ test. 
mune $\mathrm{T}$ cells demonstrates that apelin loss does not modify $\mathrm{H}-\mathrm{Y}$ alloantigen peptide-specific T lymphocyte expansion or differentiation to produce IFN- $\gamma$. These findings exclude an effect of apelin loss to increase the alloantigen burden, or enhance alloantigen presentation in mice carrying apelin-knockout grafts. However, trafficking of $\mathrm{T}$ cells to the graft and intragraft IFN- $\gamma$ expression are increased among apelin-deficient hearts.

Apelin receptor stimulation of the EC elicits PI3K and downstream eNOS activation. Apelin increases nitric oxide availability in the angiotensin-stimulated aortic aneurysm model, which is attributed to a direct antagonistic interaction between the angiotensin receptor and the apelin receptor (44). In in vitro coculture we find that the net effect of apelin receptor agonist stimulation decreases monocyte recruitment to an activated EC monolayer. This is consistent with findings that endothelial NO generation reduces transendothelial migration of dendritic cells (45). Cotreatment of allograft recipients with L-NAME dominated the effect of apelin-17, and elicited early graft rejection. Thus vascular endothelial apelin normally functions to indirectly guide inflammatory cells away from a repairing endothelium, but this defense response is overwhelmed under sustained alloimmune attack.

We find that treatment of the recipient mice with an apelin receptor agonist blunts development of the occlusive vasculopathy lesion in the allogeneic heart. In our experiment we began treatment at day 14 after transplantation, when the alloimmune response was established, intragraft expression of IFN- $\gamma$ was increased, and the angiogenic repair biomarkers were elevated. We reasoned that these features establish early vascular injury. Further, in a human heart transplant recipient, established vascular injury would prompt intervention, if effective treatment were available. The apelin receptor agonist acts in part to protect the graft by reducing established local graft inflammation and IFN- $\gamma$, since these features are decreased versus the saline-treated recipients. However, earlier work identified that rapid re-endothelialization reduces maladaptive repair after mechanical angioplasty injury of the endothelium (46), and in allogeneic tracheal transplants (47). Interestingly, the angiogenic repair biomarkers, including VEGF expression, are normalized in both artery and microvessels among heart allografts treated with the apelin receptor agonist, suggesting that reparative repopulation of the injured endothelium was achieved.

Remarkably, the apelin analog treatment effectively promotes vascular repair and microvascular density and decreases maladaptive intimal expansion of the coronary arteries despite upregulation of endogenous apelin production in the heart allograft. This is consistent with an earlier finding that tumor neovascularization is hyperinduced by cancer cell overexpression of apelin (48). The finding suggests that the induced native apelin production or availability in the setting of chronic immune injury remains rate-limiting for vascular repair. The apelin analog used in our experiments is resistant to endogenous proteinases, specifically angiotensin-converting enzyme 2 and neprilysin, that degrade and may limit angiogenic responses elicited by the native apelin peptide (30). Optimization of the stability and delivery of the analog, or development of small-molecule apelin receptor agonists, is needed for treatment of humans.

It will be important in future studies to examine whether our findings in this $\mathrm{H}-\mathrm{Y}$ minor histocompatibility model also occur in the setting of an major histocompatibility complex-mismatched transplant. The H-Y model does not reliably generate alloantibody capable of binding the surface of donor cells (49). Notably, however, the $\mathrm{H}-\mathrm{Y}$ model does include the direct and the indirect $\mathrm{T}$ cell response against the donor $(50,51)$, responses thought to play a major role in chronic rejection $(31,52,53)$.

Our experiments reveal that apelin receptor signaling mediates complex interactions between vessel wall cells and the immune system. Apelin induction in reparative vascular ECs acts in a paracrine fashion to support arterial re-endothelialization. In parallel, apelin cues the endothelium to repel mononuclear cell adhesion and invasion of the injured tissue. These functions are rate-limiting for successful repair, since pharmacological treatment with the apelin receptor agonist markedly suppresses inflammation, leukocyte-dependent IFN- $\gamma$ effects, and maladaptive intimal scarring. Apelin agonists may be an important treatment for an otherwise untreatable, fatal disease in heart transplant recipients. Further, the approach is relevant to autoimmune and mechanical arterial injuries, since repair mechanisms are likely to overlap.

\section{Methods}

Reagents. Primary antibodies are shown in Supplemental Table 1. The $\mathrm{ABC}$ kit and ImmPACT DAB peroxidase were from Vector Laboratories (catalog PK4000 and VECTSK4105). H-Y I-A $\mathrm{A}^{\mathrm{b}}$ tetramer with the peptide sequence NAGFNSNRANSSRSS was synthesized by the NIH Tetramer Core Facility (Emory University, Atlanta, Georgia, USA; http://tetramer.yerkes.emory.edu). QuantiTect mouse primers (Hprt1, Apln, Esm1, Ifng, Tnf) were purchased from Qiagen (catalog QT00166768, QT00111762, QT00129297, QT01038821, QT00104006). Other primer sets (Integrated DNA Technologies) are shown in Supplemental Tables 2 and 3. Diphenyleneiodonium chloride was from Sigma-Aldrich (catalog 300260). EasySep human monocyte isolation kit was from STEMCELL Technologies (catalog 19059). CMF019 was from Aobious (catalog AOB8242). $N_{\omega}$-NitroL-arginine methyl ester hydrochloride (L-NAME) was from SigmaAldrich (catalog N5751).

Human umbilical vein ECs (HUVECs) were isolated and cultured as described previously under approval from the Human Research Ethics Board of the University of Alberta (54). M199, DMEM, FBS, HBSS, and EC growth supplement were from Invitrogen. Recombinant human TNF- $\alpha$, VEGF-A ${ }^{165}$, and CXCL12 were from PeproTech (catalog 300-01A, 100-20, and 300-28A). Hiperfect, AllStars scrambled control siRNA (catalog SI03650318), and apelin receptor siRNA (catalog SI00073157) were from Qiagen. NMe-Apelin-17 was synthesized by J. Vederas (30). Where indicated, mice were treated with 1.5 $\mu \mathrm{mol} / \mathrm{kg} / \mathrm{d}$ via i.p. injection.

Mice and heart transplantation. WT male and female recipient C57BL/6 mice were purchased from The Jackson Laboratory (catalog 000664). The apelin-deficient (Apln $\left.n^{-/ y}\right)$ male and WT male littermate donor mice were bred on a C57BL/6 background at the University of Alberta as previously described (55). Mouse genotype was confirmed by PCR and Southern blot.

The mice underwent surgery at 11-14 weeks age. Donor hearts were transplanted heterotopically to the abdomen of the female WT recipients under isoflurane anesthesia as previously described (56). Briefly, the inferior and superior vena cava and the pulmonary vein of the donor heart were ligated. The donor aorta and pulmonary artery were anasto- 
mosed to the recipient's abdominal aorta and inferior vena cava, below the renal arteries. The donor heartbeat was assessed daily. The heart grafts were harvested when the heart allograft heartbeat was lost, or per protocol in a blinded fashion 2 or 6 weeks after transplantation.

$A p l n^{+/ y}$ heart-grafted female C57BL/6J mice were treated with apelin-17 $(1.5 \mu \mathrm{mol} / \mathrm{kg} / \mathrm{d})$ alone or in combination with L-NAME $(400 \mu \mathrm{g} / \mathrm{g} / \mathrm{d})(57)$ through daily i.p. injection, starting at week 2 after transplantation.

Left coronary artery microdissection. At the time of harvest the heart graft was fixed with 2 micro-pins in a silicon-coated dish in saline and visualized with a Zeiss Stemi 2000 Microscope. Using a microdissecting scissor and forceps, the proximal left coronary and left anterior descending artery was isolated and retrieved without surrounding tissue. The artery was placed immediately in $1 \mathrm{~mL}$ RNAlater, frozen in liquid nitrogen, then stored at $-80^{\circ} \mathrm{C}$ for later RNA extraction.

Histological processing, staining, and image analysis. The heart was sectioned in thirds. The heart base was processed for histomorphometric evaluation in $\mathrm{Zn}$ fixative. The middle third was mounted in OCT medium for frozen sections. The apex of the heart was preserved in RNAlater. The base underwent paraffin embedding, sectioning, and staining. Five-micrometer sections were taken at 100- $\mu \mathrm{m}$ levels of myocardium, and then stained using the van Gieson elastin stain to identify the internal elastic lamina. Unstained slides were used for immunohistochemistry or immunofluorescence staining on paraffin-embedded or frozen sections for CD31, F4/80, ESM1, EGFL7, CD8a, CD4, CD3, Ym1, Mac-2, and apelin according to the manufacturer's recommendations. The negative controls for antibody specificity were done omitting the primary antibody, and showed no staining. Conventional images were taken using an Olympus BX53 microscope with a top-mounted Infinity camera and Infinity Analyzer software. Immunofluorescence images were taken using a Leica DM IRB Microscope and Open Lab software. Confocal microscopy images were taken with a WaveFX microscope from Quorum Technologies with Olympus IX-81 motorized base and Yokagawa CSU 10 spinning disk confocal scan head.

To quantitate CAV lesions, regions of interest were drawn at the internal elastic lamina and the lumen of at least 3 coronary arteries in cross section per specimen using ImageJ software (NIH). The mean fractional area of intima was calculated. Endothelial gaps as a fraction of the arterial lumen circumference, microvasculature quantitation using point grids, inflammatory cell number, and the corrected total fluorescence of ESM1, apelin, and EGFL7 were each calculated using ImageJ. Colocalization index quantitation was done using the Coloc2 plug-in of Fiji software. An average of 3 images per section were analyzed.

Human heart transplant tissue. Human heart tissue was obtained under protocols approved by the Human Research Ethics Board of the University of Alberta. Human heart samples were obtained from patients undergoing transplantation for end-stage heart disease $(n$ $=6$ transplant vasculopathy, $n=6$ ischemic cardiomyopathy) at the Mazankowski Alberta Heart Institute. Transplant vasculopathy myocardium and left coronary arterial specimens were from hearts transplanted for a median 16.5 years (range 5-24 years). Nonfailing control hearts were collected from 6 consecutive organ donors whose hearts were not used because of medical or technical issues. All myocardium samples from the left ventricular free wall were collected avoiding fibrotic areas and epicardial fat. The samples were snap-frozen in liquid nitrogen and stored at $-80^{\circ} \mathrm{C}$. Fourteen archival formalin-fixed paraffin-embedded (FFPE) right ventricular endomyocardial biopsies from human heart transplant patients were retrieved from the pathology archive at the University of Alberta Hospital. These included 1 biopsy each from 9 patients with at least grade $1 \mathrm{CAV}$, diagnosed by coronary angiography and/or intravascular ultrasound, as well as 1 biopsy each from 5 patients without evidence of CAV taken at least 5 years after transplantation. Only the latest available biopsy per patient was used. Six additional FFPE right ventricular endomyocardial biopsies were also obtained from unutilized nonfailing human donor hearts from individuals with noncardiac causes of death. The 20 human endomyocardial biopsies were used for NanoString gene expression analysis, as previously described (58). Briefly, 3 consecutive $20-\mu \mathrm{m}$ sections were obtained from each FFPE block, and RNA was isolated using the RNeasy FFPE Kit (Qiagen). RNA concentration and purity were measured with a NanoDrop 2000c spectrophotometer (Thermo Fisher Scientific). Oligonucleotide probes for 4 endothelial tip cell-related genes (APLN, EGFL7, ESM1, PDGFB) and 3 housekeeping genes (LDHA, HPRT1, GAPDH) were manufactured (Integrated DNA Technologies). Gene expression was then quantified using the NanoString nCounter system (NanoString Technologies). Quality control assessment and data normalization were performed using nSolver Analysis Software version 4.0 (NanoString Technologies).

Quantitative reverse transcriptase PCR. The mouse heart apex, microdissected coronary artery, or human heart explant myocardium specimens were placed in $1 \mathrm{~mL}$ cold Trizol (Thermo Fisher Scientific) in an RNase-free tube. The samples were homogenized using a TissueLyser 2 (Qiagen) for 3 minutes at a frequency of 33 cycles per second. The RNA was extracted from the homogenates using the RNeasy Micro Kit (Qiagen) according to the manufacturer's protocol. A total of 300 ng of RNA was used to synthesize cDNA with a QuantiTect Reverse Transcription Kit (Qiagen). The primers for human and mouse genes are listed in Supplemental Tables 2 and 3. Quantitative reverse transcriptase PCR (qRT-PCR) was done using a $7500 \mathrm{ABI}$ Thermocycler. The final components of each sample reaction were as follows: $1 \mu \mathrm{L}$ of cDNA, $1 \mu \mathrm{L}$ of $10 \mu \mathrm{M}$ QuantiTect mouse/human primer sets, and $10 \mu \mathrm{L}$ of SYBR(R) Select Master Mix (Applied Biosystems) in a total volume of $20 \mu \mathrm{L}$. Samples were normalized to the internal control (mouse Hprt1 or human GAPDH) and then to normal mouse heart samples or discarded human donor hearts. Fold changes were calculated based on the $2^{-\triangle \Delta C T}$ method.

Flow cytometry. Splenocytes were harvested in some experiments for tetramer staining. Recipient cells were stained after incubation with an FcR block. Fluorophore-labeled antibodies against mouse TCR- $\beta$ chain (TCR $\beta$; H57-597), CD4 (GK1.5), CD8 $\beta$ (53-6.7), and CD45R (B220; RA3-6B2) were used.

To identify alloimmune lymphocytes by tetramer staining, $1 \times 10^{6}$ cells from spleen reconstituted in $50 \mu \mathrm{L}$ complete DMEM culture medium were incubated with $50 \mu \mathrm{L} \mathrm{Fc}$ block cocktail for 15 minutes at room temperature. The cell suspension was then stained with $42 \mu \mathrm{g} /$ mL PE-labeled I-A ${ }^{\mathrm{b}}-\mathrm{H}-\mathrm{Y}$ tetramers (catalog 34784; NAGFNSNRANSSRSS) or I-A ${ }^{\mathrm{b}}$-hCLIP tetramers (catalog 34785; PVSKMRMATPLLMQA) for 3 hours at $37^{\circ} \mathrm{C}$ with $5 \% \mathrm{CO}_{2}$ in the incubator. Cells were subsequently stained for surface markers, i.e., CD4 (RM4-5), CD8 $\beta$ (53-6.7), B220 (RA3-6B2), CD45.1 (A20), and CD44 (IM7), for 30 minutes at room temperature in the dark. Live/Dead Fixable Yellow Dead Cell Stain Kit (catalog L34959, Thermo Fisher Scientific) was used to exclude dead cells. 
For tetramer and intracellular cytokine staining, splenic cells were enriched for CD4 T cells using EasySep mouse CD4 ${ }^{+} \mathrm{T}$ cell isolation kit (catalog 19752, STEMCELL Technologies). Enriched populations were then reconstituted in $50 \mu \mathrm{L}$ complete DMEM culture medium and incubated with $50 \mu \mathrm{L} \mathrm{Fc}$ block cocktail at $37^{\circ} \mathrm{C}$ with $5 \% \mathrm{CO}_{2}$ in the incubator with PMA $(20 \mathrm{ng} / \mathrm{mL})$ plus ionomycin $(1 \mu \mathrm{g} / \mathrm{mL})$ for a total of 5 hours. Brefeldin A $(3 \mu \mathrm{g} / \mathrm{mL})$ and monensin $(2 \mu \mathrm{M})$ were added 1 hour after PMA/ionomycin. The mixture was then stained with $42 \mu \mathrm{g} / \mathrm{mL}$ tetramers for the last 3 hours in the incubator. Cells were subsequently stained for surface markers for 30 minutes at room temperature in the dark. Dead cells were excluded using the Live/ Dead Fixable Yellow Dead Cell Stain Kit. Cells were then fixed and permeabilized with eBioscience IC fixation (catalog 00-8222-49) and permeabilization buffer (catalog 00-8222), and stained with antibodies for IFN- $\gamma$ (XMG1.2). Fc block cocktail was a mixture of 3 $\mathrm{mL}$ each of normal mouse, rat, and hamster serum, with addition of $0.3 \mathrm{mg}$ of anti-CD16/32 antibody (clone 2.4g2, BioXCell). Complete DMEM contains Dulbecco's modified Eagle medium (DMEM), 10\% FBS, $2 \mathrm{mM}$ L-glutamine, $1 \mathrm{mM}$ MEM sodium pyruvate, $100 \mu \mathrm{M}$ nonessential amino acids, $100 \mathrm{U} / \mathrm{mL}$ penicillin, $100 \mathrm{U} / \mathrm{mL}$ streptomycin, $50 \mu \mathrm{M} 2$-mercaptoethanol. All fluorophore-labeled antibodies for flow cytometry, except tetramers, were purchased from eBioscience. BD LSR II (BD Biosciences) was used for the data acquisition. Flow cytometric data analysis was performed using FlowJo (Tree Star Software). Gating strategies are shown in Supplemental Figure 10.

Cell culture. HUVECs were isolated as previously described, and used below passage 5 (54). Human peripheral blood mononuclear cells were isolated from whole blood using Lymphoprep according to the manufacturer's protocol. The mononuclear cell fraction was harvested, washed, and treated with RBC lysis buffer (00-4333-57, Invitrogen). Monocytes were isolated by negative selection (catalog 19059, STEMCELL Technologies). The purity of the isolated monocyte population was greater than $95 \%$ by flow cytometry.

Monocyte adhesion assay. Confluent HUVEC monolayers were pretreated with TNF- $\alpha(100 \mathrm{ng} / \mathrm{mL})$ for 18 hours, and then human monocytes were added in 1:10 ratio. The cocultures were mock-treated, or treated with an apelin-17 receptor agonist $(1 \mu \mathrm{M})$ or DPE NOS3 inhibitor $(180 \mathrm{nM})$ as indicated. The wells were washed twice with warm M199, and then adherent monocytes in 5 random fields per well were counted in each experiment.

Scratch wound assay. HUVECs were transfected with apelin receptor or scrambled siRNA, or mock-transfected, then plated at confluence. The HUVEC monolayers were wounded with the tip of a $200-\mu \mathrm{L}$ pipette, then cultured at $37^{\circ} \mathrm{C}$ in M199/2\% FBS with VEGF-A ${ }^{165}(50 \mathrm{ng} / \mathrm{mL})$, or apelin-17 receptor agonist alone $(1 \mu \mathrm{M})$ or in combination as indicated. Serial images were captured at $0,4,8$, or 10 hours in each experiment. Quantitation of the wound area was done using Image J software.

RNA interference. HUVECs were seeded in a 6-well plate at 50\%$60 \%$ confluence, then transfected twice over 2 days with either $50 \mathrm{nM}$ nonspecific siRNA (siNS) or specific apelin receptor siRNA using Hiperfect per the manufacturer's protocol. Cells were studied 2 days after the second transfection.

Western blots. Cell monolayers were washed once with ice-cold $1 \times$ PBS, then lysed using ice-cold RIPA buffer (10 mM Tris, pH 7.4, $100 \mathrm{mM}$ $\mathrm{NaCl}, 1 \mathrm{mM}$ EDTA, $1 \mathrm{mM}$ EGTA, $5 \mathrm{mM} \mathrm{NaF}, 2 \mathrm{mM} \mathrm{Na}_{3} \mathrm{VO}_{4}, 0.1 \%$ SDS, $0.5 \%$ Na deoxycholate, $1 \%$ Triton X-100, 10\% glycerol, 1 mM PMSF) and boiled at $95^{\circ} \mathrm{C}$ for 5 minutes. Proteins were separated on SDS-PAGE, transferred to a nitrocellulose membrane (Bio-Rad), and blocked with $5 \%$ skimmed milk in $1 \times$ TBS plus $0.1 \%$ Tween-20. The membranes were immunoblotted overnight at $4^{\circ} \mathrm{C}$ with the desired antibodies (diluted in $5 \%$ BSA), washed 3 times in $1 \times$ TBST, incubated with the secondary antibodies (diluted in $5 \%$ milk), washed 3 times in $1 \times$ TBST, then visualized using an Odyssey infrared scanner (Licor). Protein bands were equally contrast-enhanced with Adobe Photoshop CS3 and quantified with Image Studio Lite software (LI-COR Biosciences.

In vitro $3 D$ angiogenesis beads assay. Evaluation of in vitro angiogenesis was performed as previously described (59). Briefly, HUVECs were labeled with CellTracker Green (Life Technologies). Cytodex beads were coated with HUVECs ( 400 cells per bead) and cultured for 4 hours in M199, with 10\% FBS, and $20 \mathrm{ng} / \mathrm{mL}$ VEGF. The beads were washed twice, suspended in fibrinogen $(2 \mathrm{mg} / \mathrm{mL})$ with aprotinin $(0.15 \mathrm{U} / \mathrm{mL})$, and $0.625 \mathrm{U} / \mathrm{mL}$ thrombin was added. To study the effect of apelin treatment on vascular EC sprouting and induction of tip cell differentiation, apelin-17 (300 or $1000 \mathrm{nM}$ ) and/ or VEGF $(15 \mathrm{ng} / \mathrm{mL}$ ) were added with the growth medium on top of each well. Endothelial cell growth supplement was used as a positive control for EC sprouting. All treated wells were left incubated for 24 hours in $5 \% \mathrm{CO}_{2}$ at $37^{\circ} \mathrm{C}$.

To analyze the endothelial angiogenic sprouting, 30 beads per treatment group from each experiment were imaged, using a $\times 20$ objective lens and a CCD camera equipped with an inverted fluorescence microscope (Leica DM-IRB microscope). Scoring was done using OpenLab (PerkinElmer).

Statistics. Data sets were tested for normality by the D'Agostino and Pearson test using Prism 7 software (GraphPad). Data are presented as mean \pm SEM. Using Prism 7 software, statistical analysis was conducted by 1-way or 2-way ANOVA as appropriate followed by the Bonferroni post hoc test. Pairwise comparisons were done by unpaired 2-sided Student's $t$ test, or, in the case of the smaller data sets, the 2-sided Mann-Whitney test or paired Student's $t$ test as indicated. A $P$ value less than 0.05 was considered significant.

Study approval. Animal experiments were carried out according to the Canadian Council on Animal Care guidelines, and were approved by the Animal Care and Use Committee at the University of Alberta. Human tissue was obtained under approval of the Human Research Ethics Board at the University of Alberta.

\section{Author contributions}

A.G. Murray, BS, CCA, and GYO conceived the project. A.G. Masoud, JXL, AKA, MAF, LFZ, and BAA generated the data. DK, CF, JCV, HZ, GYO, and ZK provided key experimental reagents. A.G. Masoud, AA, MAF, JXL, CCA, BAA, RBM, and A.G. Murray interpreted the data. A.G. Masoud and A.G. Murray wrote the manuscript.

\section{Acknowledgments}

The authors thank Philip F. Halloran for critical review of the manuscript. This work is supported by a Grant-in-Aid from the Heart and Stroke Foundation of Canada (G-17-0018233, to A.G. Murray and BS). Studentship support to A.G. Masoud was from the University of Alberta Hospital Foundation.

Address correspondence to: Allan G. Murray, Room 275 HMRC, University of Alberta, Edmonton, Alberta, Canada T6G 2S2. Phone: 780.407.8741; Email: allan.murray@ualberta.ca. 
1. Lund LH, et al. The Registry of the International Society for Heart and Lung Transplantation: Thirty-second Official Adult Heart Transplantation Report-2015; Focus Theme: Early graft failure. J Heart Lung Transplant. 2015;34(10):1244-1254.

2. Lund LH, et al. The Registry of the International Society for Heart and Lung Transplantation: Thirty-fourth Adult Heart Transplantation Report -2017; Focus Theme: Allograft ischemic time. J Heart Lung Transplant. 2017;36(10):1037-1046.

3. Pober JS, Jane-wit D, Qin L, Tellides G. Interacting mechanisms in the pathogenesis of cardiac allograft vasculopathy. Arterioscler Thromb Vasc Biol. 2014;34(8):1609-1614.

4. Loupy A, et al. Late failing heart allografts: pathology of cardiac allograft vasculopathy and association with antibody-mediated rejection. Am J Transplant. 2016;16(1):111-120.

5. Choy JC, Kerjner A, Wong BW, McManus BM, Granville DJ. Perforin mediates endothelial cell death and resultant transplant vascular disease in cardiac allografts. Am J Pathol. 2004;165(1):127-133.

6. Lin CM, Plenter RJ, Coulombe M, Gill RG. Interferon gamma and contact-dependent cytotoxicity are each rate limiting for natural killer cellmediated antibody-dependent chronic rejection. Am J Transplant. 2016;16(11):3121-3130.

7. Nagano H, Mitchell RN, Taylor MK, Hasegawa S, Tilney NL, Libby P. Interferon- $\gamma$ deficiency prevents coronary arteriosclerosis but not myocardial rejection in transplanted mouse hearts. J Clin Invest. 1997;100(3):550-557.

8. Uehara S, et al. NK cells can trigger allograft vasculopathy: the role of hybrid resistance in solid organ allografts. JImmunol. 2005;175(5):3424-3430.

9. Kitchens WH, et al. Macrophage depletion suppresses cardiac allograft vasculopathy in mice. Am J Transplant. 2007;7(12):2675-2682.

10. van Loosdregt J, et al. The chemokine and chemokine receptor profile of infiltrating cells in the wall of arteries with cardiac allograft vasculopathy is indicative of a memory T-helper 1 response. Circulation. 2006;114(15):1599-1607.

11. Wang $\mathrm{Y}$, et al. Interferon-gamma induces human vascular smooth muscle cell proliferation and intimal expansion by phosphatidylinositol 3-kinase dependent mammalian target of rapamycin raptor complex 1 activation. Circ Res. 2007;101(6):560-569.

12. Nadig SN, et al. In vivo prevention of transplant arteriosclerosis by ex vivo-expanded human regulatory T cells. Nat Med. 2010;16(7):809-813.

13. Tellides $\mathrm{G}$, et al. Interferon- $\gamma$ elicits arteriosclerosis in the absence of leukocytes. Nature. 2000;403(6766):207-211.

14. Claesson-Welsh L, Welsh M. VEGFA and tumour angiogenesis. JIntern Med. 2013;273(2):114-127.

15. del Toro R, et al. Identification and functional analysis of endothelial tip cell-enriched genes. Blood. 2010;116(19):4025-4033.

16. Eilken HM, Adams RH. Dynamics of endothelial cell behavior in sprouting angiogenesis. Curr Opin Cell Biol. 2010;22(5):617-625.

17. Cox CM, D'Agostino SL, Miller MK, Heimark RL, Krieg PA. Apelin, the ligand for the endothelial G-protein-coupled receptor, APJ, is a potent angiogenic factor required for normal vascular development of the frog embryo. Dev Biol. 2006;296(1):177-189.

18. Charo DN, et al. Endogenous regulation of cardiovascular function by apelin-APJ. Am J Physiol Heart Circ Physiol. 2009;297(5):H1904-H1913.

19. Scimia MC, et al. APJ acts as a dual receptor in cardiac hypertrophy. Nature. 2012;488(7411):394-398.

20. Pi J, et al. Apln-CreERT:mT/mG reporter mice as a tool for sprouting angiogenesis study. BMC Ophthalmol. 2017;17(1):163.

21. Eyries M, et al. Hypoxia-induced apelin expression regulates endothelial cell proliferation and regenerative angiogenesis. Circ Res. 2008;103(4):432-440.

22. Kasai A, et al. Retardation of retinal vascular development in apelin-deficient mice. Arterioscler Thromb Vasc Biol. 2008;28(10):1717-1722.

23. Wang $\mathrm{W}$, et al. Loss of Apelin exacerbates myocardial infarction adverse remodeling and ischemia-reperfusion injury: therapeutic potential of synthetic Apelin analogues. J Am Heart Assoc. 2013;2(4):e000249.

24. Marelli-Berg FM, et al. Cognate recognition of the endothelium induces HY-specific CD8 ${ }^{+}$ T-lymphocyte transendothelial migration (diapedesis) in vivo. Blood. 2004;103(8):3111-3116.

25. Bagai $R$, et al. Mouse endothelial cells cross-present lymphocyte-derived antigen on class I MHC via a TAP1- and proteasome-dependent pathway. JImmunol. 2005;174(12):7711-7715.

26. Saint-Geniez M, Masri B, Malecaze F, Knibiehler $\mathrm{B}$, Audigier Y. Expression of the murine msr/ apj receptor and its ligand apelin is upregulated during formation of the retinal vessels. Mech Dev. 2002;110(1-2):183-186.

27. Gerhardt $\mathrm{H}$, et al. VEGF guides angiogenic sprouting utilizing endothelial tip cell filopodia. JCell Biol. 2003;161(6):1163-1177.

28. Roudnicky F, et al. Endocan is upregulated on tumor vessels in invasive bladder cancer where it mediates VEGF-A-induced angiogenesis. Cancer Res. 2013;73(3):1097-1106.

29. Villa E, et al. Neoangiogenesis-related genes are hallmarks of fast-growing hepatocellular carcinomas and worst survival. Results from a prospective study. Gut. 2016;65(5):861-869.

30. McKinnie SMK, et al. Synthetic modification within the "RPRL" region of apelin peptides: impact on cardiovascular activity and stability to neprilysin and plasma degradation. J Med Chem. 2017;60(14):6408-6427.

31. Kaul AM, et al. Acute and chronic rejection: compartmentalization and kinetics of counterbalancing signals in cardiac transplants. Am J Transplant. 2015;15(2):333-345.

32. Liu Q, et al. Donor dendritic cell-derived exosomes promote allograft-targeting immune response. JClin Invest. 2016;126(8):2805-2820.

33. Kazakov A, et al. Inhibition of endothelial nitric oxide synthase induces and enhances myocardial fibrosis. Cardiovasc Res. 2013;100(2):211-221.

34. McDonald AI, et al. Endothelial regeneration of large vessels is a biphasic process driven by local cells with distinct proliferative capacities. Cell Stem Cell. 2018;23(2):210-225.e6.

35. Levéen P, Pekny M, Gebre-Medhin S, Swolin B,
Larsson E, Betsholtz C. Mice deficient for PDGF B show renal, cardiovascular, and hematological abnormalities. Genes Dev. 1994;8(16):1875-1887.

36. Rocha SF, et al. Esm1 modulates endothelial tip cell behavior and vascular permeability by enhancing VEGF bioavailability. Circ Res. 2014;115(6):581-590.

37. Oladipupo SS, et al. Endothelial cell FGF signaling is required for injury response but not for vascular homeostasis. Proc Natl Acad Sci U S A. 2014;111(37):13379-13384.

38. Hara C, et al. Laser-induced choroidal neovascularization in mice attenuated by deficiency in the apelin-APJ system. Invest Ophthalmol Vis Sci. 2013;54(6):4321-4329.

39. Hashimoto T, et al. Requirement of apelin-apelin receptor system for oxidative stress-linked atherosclerosis. Am J Pathol. 2007;171(5):1705-1712.

40. Kojima Y, et al. Upregulation of the apelin-APJ pathway promotes neointima formation in the carotid ligation model in mouse. Cardiovasc Res. 2010;87(1):156-165.

41. Hosaka K, et al. Pericyte-fibroblast transition promotes tumor growth and metastasis. Proc Natl Acad Sci U S A. 2016;113(38):E5618-E5627.

42. Malabanan KP, Sheahan AV, Khachigian LM. Platelet-derived growth factor-BB mediates cell migration through induction of activating transcription factor 4 and tenascin-C. Am J Pathol. 2012;180(6):2590-2597.

43. Soncin F, et al. VE-statin, an endothelial repressor of smooth muscle cell migration. EMBO J. 2003;22(21):5700-5711.

44. Chun HJ, et al. Apelin signaling antagonizes Ang II effects in mouse models of atherosclerosis. JClin Invest. 2008;118(10):3343-3354

45. Weis M, Schlichting CL, Engleman EG, Cooke JP. Endothelial determinants of dendritic cell adhesion and migration: new implications for vascular diseases. Arterioscler Thromb Vasc Biol. 2002;22(11):1817-1823.

46. Asahara T, et al. Local delivery of vascular endothelial growth factor accelerates reendothelialization and attenuates intimal hyperplasia in balloon-injured rat carotid artery. Circulation. 1995;91(11):2793-2801.

47. Jiang X, et al. Adenovirus-mediated HIF- $1 \alpha$ gene transfer promotes repair of mouse airway allograft microvasculature and attenuates chronic rejection. JClin Invest. 2011;121(6):2336-2349.

48. Sorli SC, Le Gonidec S, Knibiehler B, Audigier Y. Apelin is a potent activator of tumour neoangiogenesis. Oncogene. 2007;26(55):7692-7699.

49. Simpson E, Scott D, Chandler P. The malespecific histocompatibility antigen, H-Y: a history of transplantation, immune response genes, sex determination and expression cloning. Annu Rev Immunol. 1997;15:39-61.

50. Chen Y, Demir Y, Valujskikh A, Heeger PS. The male minor transplantation antigen preferentially activates recipient $\mathrm{CD} 4+\mathrm{T}$ cells through the indirect presentation pathway in vivo. J Immunol. 2003;171(12):6510-6518.

51. Valujskikh A, Lantz O, Celli S, Matzinger P, Heeger PS. Cross-primed CD8(+) T cells mediate graft rejection via a distinct effector pathway. Nat Immunol. 2002;3(9):844-851.

52. Chen Y, Demir Y, Valujskikh A, Heeger PS. Anti- 
gen location contributes to the pathological features of a transplanted heart graft. Am J Pathol. 2004;164(4):1407-1415.

53. Chan WF, Razavy H, Anderson CC. Differential susceptibility of allogeneic targets to indirect CD4 immunity generates split tolerance. J Immunol. 2008;181(7):4603-4612.

54. Mullaly SC, Moyse RJ, Nelson RC, Murray AG. Stable lymphocyte contact induces remodeling of endothelial cell matrix receptor complexes.
Eur J Immunol. 2002;32(5):1493-1501.

55. Kuba K, et al. Impaired heart contractility in Apelin gene-deficient mice associated with aging and pressure overload. Circ Res. 2007;101(4):e32-e42.

56. Russell PS, et al. Coronary artery disease from isolated non-H2-determined incompatibilities in transplanted mouse hearts. Transplantation. 2011;91(8):847-852.

57. Peotta VA, Vasquez EC, Meyrelles SS. Cardiovascular neural reflexes in L-NAME-induced hypertension in mice. Hypertension. 2001;38(3 pt 2):555-559.

58. Adam B, et al. Multiplexed color-coded probebased gene expression assessment for clinical molecular diagnostics in formalin-fixed paraffinembedded human renal allograft tissue. Clin Transplant. 2016;30(3):295-305.

59. Nakatsu MN, Hughes CC. An optimized threedimensional in vitro model for the analysis of angiogenesis. Meth Enzymol. 2008;443:65-82. 